FACTA UNIVERSITATIS (NIŠ)

Ser. Math. Inform. Vol. 36, No 2 (2021), 259-274

https://doi.org/10.22190/FUMI200602020G

Original Scientific Paper

\title{
PARANORMED SPACES OF ABSOLUTE LUCAS SUMMABLE SERIES AND MATRIX OPERATORS
}

\author{
Fadime Gökçe \\ University of Pamukkale, Faculty of Science, \\ Department of Mathematics, Denizli, Turkey
}

\begin{abstract}
The aim of this paper is to introduce the absolute series space $\left|\mathcal{L}^{\phi}(r, s)\right|(\mu)$ as the set of all series summable by the absolute Lucas method, and to give its topological and algebraic structure such as $F K$-space, duals and Schauder basis. Also, certain matrix operators on this space are characterized.

Keywords: Absolute summability, Lucas numbers, matrix transformations, Maddox space, sequence spaces, bounded operators
\end{abstract}

\section{Introduction}

Let $\omega$ be the set of all sequences of complex numbers. Any vector subspace of $\omega$ is called a sequence space. We write $c, l_{\infty}, \Psi$ for the spaces of all convergent, bounded and finite sequences, and also write $c s, b s$ and $l_{p}(p \geq 1)$ for the spaces of all convergent, bounded, $p$-absolutely convergent series, respectively.

Let $X$ and $Y$ be two sequence spaces and $A=\left(a_{n v}\right)$ be an arbitrary infinite matrix of complex numbers. If a series

$$
A_{n}(x)=\sum_{v=0}^{\infty} a_{n v} x_{v}
$$

converges for all $n \in \mathbb{N}=\{0,1,2, \ldots\}$, then, by $A(x)=\left(A_{n}(x)\right)$, we denote the $A$-transform of the sequence $x=\left(x_{v}\right)$. Also, if $A x=\left(A_{n}(x)\right) \in Y$ for every $x \in X$,

Received June 2, 2020, accepted: November 24, 2020

Communicated by Dijana Mosić

Corresponding Author: Fadime Gökçe, University of Pamukkale, Faculty of Science, Department

of Mathematics, Denizli, Turkey | E-mail: fgokce@pau.edu.tr

2010 Mathematics Subject Classification. Primary 40C05, 11B39, 40F05, 46A45

(C) 2021 by University of Niš, Serbia | Creative Commons License: CC BY-NC-ND 
we say that $A$ defines a matrix transformation from $X$ to $Y$, and by $(X, Y)$ denote the class of all infinite matrices from $X$ into $Y$. The set

$$
S(X, Y)=\left\{a=\left(a_{v}\right) \in \omega: a x=\left(a_{k} x_{k}\right) \in Y \text { for all } x \in X\right\}
$$

is called the multiplier space of $X$ and $Y$. According to this notation, the $\alpha-, \beta$ and $\gamma$ - duals of the space $X$ are identified as

$$
X^{\alpha}=S(X, l), X^{\beta}=S(X, c s), X^{\gamma}=S(X, b s) .
$$

The concept of the domain of an infinite matrix $A$ in the sequence space $X$ is given by

$$
X_{A}=\left\{x=\left(x_{n}\right) \in \omega: A(x) \in X\right\} .
$$

Using the concept of the matrix domain, several sequence spaces have been introduced and their algebraic, topological structure and matrix transformations have been studied in literature (see $[1,2,4,10,13,14,15,16]$ ).

If $a_{n n} \neq 0$ for all $n$ and $a_{n v}=0$ for $n<v$, then $A$ is called a triangle matrix. The matrix domains of triangles have an important role in literature. For example, if $A$ is a triangle and $X$ is an $F K$-space, a complete locally convex linear metric space with continuous coordinates $p_{n}: X \rightarrow \mathbb{C}$ defined by $p_{n}(x)=x_{n}$ for all $n \in \mathbb{N}$, then the sequence space $X_{A}$ is also an $F K$-space [11]. If there exists unique sequence of coefficients $\left(x_{k}\right)$ such that, for each $x \in X$,

$$
\lim _{m \rightarrow \infty} \sum_{k=0}^{m} x_{k} b_{k}=x
$$

then, the sequence $\left(b_{k}\right)$ is called the Schauder basis (or briefly basis) for a sequence space $X$. For instance, the sequence $\left(e^{(j)}\right)$ is the Schauder basis of the space $l_{p}$, where $e^{(j)}$ is the sequence whose only non-zero term is 1 in $j$ th place for each $j \in \mathbb{N}$, [23].

The following result is useful to find a Schauder basis for the matrix domain of a special triangular matrix in a linear metric space.

Lemma 1.1. ([11]). If $\left(b_{k}\right)$ is a Schauder basis of the metric space $(X, d)$, then $\left(S\left(b_{k}\right)\right)$ is a basis of $X_{T}$ with respect to the metric $d_{T}$ given by $d_{T}\left(z_{1}, z_{2}\right)=d\left(T z_{1}\right.$, $\left.T z_{2}\right)$ for all $z_{1}, z_{2} \in X_{T}$, where $T$ is a triangular matrix and $S$ is its inverse.

The well known space $l(\mu)$ of Maddox is defined by

$$
l(\mu)=\left\{x=\left(x_{n}\right): \sum_{n=1}^{\infty}\left|x_{n}\right|^{\mu_{n}}<\infty\right\},
$$

which is an $F K$-space with $A K$ with respect to its natural paranorm

$$
g(x)=\left(\sum_{n=0}^{\infty}\left|x_{n}\right|^{\mu_{n}}\right)^{1 / M},
$$


where $M=\max \left\{1, \sup _{n} \mu_{n}\right\}$; also it is even a $B K$-space if $\mu_{n} \geq 1$ for all $n$ with respect to the norm

$$
\|x\|=\inf \left\{\delta>0: \sum_{n=0}^{\infty}\left|x_{n} / \delta\right|^{\mu_{n}} \leq 1\right\},
$$

$([18,19,20])$.

Throughout the paper, we suppose that $0<\inf \mu_{n} \leq H<\infty$ and $\mu_{n}^{*}$ is conjugate of $\mu_{n}$, that is, $1 / \mu_{n}+1 / \mu_{n}^{*}=1$ for $\mu_{n}>1$, and $1 / \mu_{n}^{*}=0$ for $\mu_{n}=1$, for all $n \in \mathbb{N}$.

Let $\sum x_{v}$ be an infinite series with $n$th partial sum $s_{n},\left(\phi_{n}\right)$ be a sequence of positive numbers and $\left(\mu_{n}\right)$ be a bounded sequence of positive numbers. Then, the series $\sum x_{v}$ is said to be summable $\left|A, \phi_{n}\right|(\mu)$, if

$$
\sum_{n=0}^{\infty} \phi_{n}^{\mu_{n}-1}\left|\Delta A_{n}(s)\right|^{\mu_{n}}<\infty,
$$

where $\Delta A_{n}(s)=A_{n}(s)-A_{n-1}(s), A_{-1}(s)=0,[6]$.

Note that, $\left|A, \phi_{n}\right|(\mu)$ includes many well known methods; if $A$ is the matrix of weighted mean $\left(\bar{N}, p_{n}\right)$ (resp. $\left.\phi_{n}=P_{n} / p_{n}\right)$ with $\mu_{n}=k$ for all $n$, then it reduces to the summability $\left|\bar{N}, p_{n}, \phi_{n}\right|_{k}[29]$ (the summability $\left|\bar{N}, p_{n}\right|_{k}[3]$ ). Also, if we take $A$ as the matrix of Cesàro mean of order $\alpha>-1$ and $\phi_{n}=n$ with $\mu_{n}=k$ for all $n$, then we get the summability $|C, \alpha|_{k}$ in Flett's notation [5].

In addition to the aforementioned spaces, some absolute series spaces have also been studied in the literature (see $[6,7,8,9,11,25,27]$ ).

One of the main purposes of this paper is to define a new series space $\left|\mathcal{L}^{\phi}(r, s)\right|(\mu)$ as the set of all series summable by the absolute Lucas matrix method and investigate its topological and algebraic structures. Also, by means of a given basic lemma, we characterize certain matrix operators on this space.

\section{Absolute Lucas Series Space $\left|\mathcal{L}^{\phi}(r, s)\right|(\mu)$}

In this section, we will first remind you of some properties of Lucas numbers. The Lucas sequence $\left(L_{n}\right)$ is one of the most interesting number sequence in mathematics and it is named after the mathematician François Edouard Anatole Lucas (18421891). The $n$th Lucas number $L_{n}$ is given by the Fibonacci recurrence relation with different initial condition such that

$$
L_{0}=2, L_{1}=1 \text { and } L_{n}=L_{n-1}+L_{n-2} \text { for } n \geq 2,
$$

which also has some interesting relations as follows

$$
\sum_{k=1}^{n} L_{k}=L_{n+2}-3, \sum_{k=1}^{n} L_{2 k-1}=L_{2 n}-2
$$




$$
\begin{gathered}
\sum_{k=1}^{n} L_{2 k}=L_{2 n+1}-1, \quad \sum_{k=1}^{n} L_{k}^{2}=L_{n} L_{n+1}-2 \\
L_{n-1}^{2}+L_{n} L_{n-1}-L_{n}^{2}=5(-1)^{n+1}, n \geq 1 \\
L_{n-1} L_{n+1}-L_{n}^{2}=5(-1)^{n+1}, n \geq 1 .
\end{gathered}
$$

We refer reader to [17] for other properties of these numbers. In addition to all these features, just like the Fibonacci numbers, the rate of successive Lucas numbers converges to the golden ratio which is one of the most interesting irrational having an important role in number theory, algorithms, network theory, etc.

Recently, using Lucas numbers, the Lucas matrix $\hat{E}(r, s)=\left(\hat{e}_{n k}(r, s)\right)$ has been defined by

$$
\hat{e}_{n k}(r, s)=\left\{\begin{array}{lr}
s \frac{L_{n}}{L_{n-1}}, & k=n-1 \\
r \frac{L_{n-1}}{L_{n}}, & k=n \\
0, & \text { otherwise }
\end{array}\right.
$$

where $L_{n}$ be the $n$th Lucas number for every $n \in \mathbb{N}$ and $r, s \in \mathbb{R} \backslash\{0\}$ [15].

We are now ready to establish and study the series space $\left|\mathcal{L}^{\phi}(r, s)\right|(\mu)$. Put the Lucas matrix instead of $A$ in (1.2), then $\left|A, \phi_{n}\right|(\mu)$ summability is reduced to the absolute Lucas summability, i.e.,

$$
\sum_{n=0}^{\infty} \phi_{n}^{\mu_{n}-1}\left|\Delta \hat{E}_{n}(r, s)\right|^{\mu_{n}}<\infty
$$

So, we introduce the space $\left|\mathcal{L}^{\phi}(r, s)\right|(\mu)$ by the set of all series satisfying the condition (2.1). Also, since $\left(s_{n}\right)$ is the sequence of partial sums of the series $\sum x_{k}$, it can be written that

$$
\begin{aligned}
\hat{E}_{n}(r, s)=\sum_{v=1}^{n} \hat{e}_{n v}(r, s) s_{v} & =\sum_{k=1}^{n} x_{k} \sum_{v=k}^{n} \hat{e}_{n v}(r, s) \\
& =x_{n} \hat{e}_{n n}(r, s)+\sum_{k=1}^{n-1}\left(\hat{e}_{n n}(r, s)+\hat{e}_{n, n-1}(r, s)\right) x_{k} \\
& =x_{n} r \frac{L_{n-1}}{L_{n}}+\sum_{k=1}^{n-1}\left(s \frac{L_{n}}{L_{n-1}}+r \frac{L_{n-1}}{L_{n}}\right) x_{k} \\
& =\sum_{k=1}^{n} l_{n k} x_{k}
\end{aligned}
$$

where $\mathcal{L}(r, s)=\left(l_{n k}\right)$ is the matrix given by

$$
l_{n k}=\left\{\begin{array}{lr}
r \frac{L_{n-1}}{L_{n}}, & k=n \\
s \frac{L_{n}}{L_{n-1}}+r \frac{L_{n-1}}{L_{n}}, & 1 \leq k \leq n-1 \\
0, & k>n .
\end{array}\right.
$$


Hence we get

$$
\begin{aligned}
\Delta \hat{E}_{n}(r, s)= & r \frac{L_{n-1}}{L_{n}} x_{n}+\left(s \frac{L_{n}}{L_{n-1}}+r \frac{5(-1)^{n+1}}{L_{n} L_{n-1}}\right) x_{n-1} \\
& +\sum_{k=1}^{n-2} \frac{5(-1)^{n}}{L_{n-1}}\left(\frac{s}{L_{n-2}}-\frac{r}{L_{n}}\right) x_{k} \\
= & \sum_{k=1}^{n} \xi_{n k} x_{k},
\end{aligned}
$$

where

$$
\xi_{n k}=\left\{\begin{array}{lr}
r \frac{L_{n-1}}{L_{n}}, & k=n \\
s \frac{L_{n}}{L_{n-1}}+r \frac{5(-1)^{n+1}}{L_{n} L_{n-1}}, & k=n-1 \\
\frac{5(-1)^{n}}{L_{n-1}}\left(\frac{s}{L_{n-2}}-\frac{r}{L_{n}}\right), & 1 \leq k<n-2 \\
0, & k>n .
\end{array}\right.
$$

This means that a series $\sum x_{k}$ is summable by the absolute Lucas method if a sequence $\left(x_{k}\right) \in\left|\mathcal{L}^{\phi}(r, s)\right|(\mu)$, i.e.,

$$
\left|\mathcal{L}^{\phi}(r, s)\right|(\mu)=\left\{x \in \omega:\left(\phi_{n}^{1 / \mu_{n}^{*}} \sum_{k=0}^{n} \xi_{n k} x_{k}\right) \in l(\mu)\right\} .
$$

Note that there is a close relation between this space and the Maddox's space. In fact, according to the concept of domain, it can be redefined by

$$
\left|\mathcal{L}^{\phi}(r, s)\right|(\mu)=(l(\mu))_{E^{(\mu)} \circ \mathcal{L}(r, s)}
$$

where $E^{(\mu)}=\left(e_{n k}^{(\mu)}\right)$ is given by

$$
e_{n k}^{(\mu)}=\left\{\begin{array}{lr}
\phi_{n}^{1 / \mu^{*}}, & k=n \\
-\phi_{n}^{1 / \mu^{*}}, & k=n-1 \\
0, & k \neq n, n-1
\end{array}\right.
$$

Also, note that

$$
\left(E^{(\mu)} \circ \mathcal{L}(r, s)\right)_{n}(x)=\phi_{n}^{1 / \mu^{*}}\left(\mathcal{L}_{n}(r, s)(x)-\mathcal{L}_{n-1}(r, s)(x)\right) .
$$

On the other hand, since every triangle matrix has a unique inverse which is also a triangle [30], the matrices $\mathcal{L}(r, s)$ and $E^{(\mu)}$ have unique inverses $\tilde{\mathcal{L}}(r, s)=\left(\tilde{l}_{n k}\right)$ and $\tilde{E}^{(\mu)}=\left(\tilde{e}_{n k}\right)$ which we have been computed as

$$
\tilde{l}_{n k}=\left\{\begin{array}{lr}
\frac{1}{r} \frac{L_{n}}{L_{n-1}}, & k=n \\
\frac{(-1)^{n-k}}{r}\left(\frac{s}{r}\right)^{n-1-k} \frac{1}{L_{k} L_{k-1}}\left(\frac{s}{r} L_{n}^{2}+L_{n-1}^{2}\right), & 1 \leq k \leq n-1 \\
0, & k>n
\end{array}\right.
$$




$$
\tilde{e}_{n k}^{(\mu)}=\left\{\begin{array}{lr}
\theta_{k}^{-1 / \mu_{k}^{*}}, & 1 \leq k \leq n \\
0, & k>n
\end{array}\right.
$$

respectively.

For the proofs of theorems we require some well known lemmas.

Lemma 2.1. ([12]) Let $\mu=\left(\mu_{v}\right)$ and $\lambda=\left(\lambda_{v}\right)$ be any two bounded sequences of strictly positive numbers.

(i) If $\mu_{v}>1$ for all $v$, then, $A \in(l(\mu), l)$ if and only if there exists an integer $M>1$ such that

$$
\sup \left\{\sum_{v=0}^{\infty}\left|\sum_{n \in K} a_{n v} M^{-1}\right|^{\mu_{v}^{*}}: K \subset \mathbb{N} \text { finite }\right\}<\infty .
$$

(ii) If $\mu_{v} \leq 1$ and $\lambda_{v} \geq 1$ for all $v \in \mathbb{N}$, then $A \in(l(\mu), l(\lambda))$ if and only if there exists some $M$ such that

$$
\sup _{v} \sum_{n=0}^{\infty}\left|a_{n v} M^{-1 / \mu_{v}}\right|^{\lambda_{n}}<\infty
$$

(iii) If $\mu_{v} \leq 1$, then, $A \in(l(\mu), c)$ if and only if

$$
\text { (a) } \lim _{n} a_{n v} \text { exists for each } v,(b) \sup _{n, v}\left|a_{n v}\right|^{\mu_{v}}<\infty \text {, }
$$

and $A \in\left(l(\mu), l_{\infty}\right)$ if $(b)$ holds.

(iv) If $\mu_{v}>1$ for all $v$, then, $A \in(l(\mu), c)$ if and only if

(a) $\lim _{n} a_{n v}$ exists for each $v,(b)$ there is a number $M>1$ such that

$$
\sup _{n} \sum_{v=0}^{\infty}\left|a_{n v} M^{-1}\right|^{\mu_{v}^{*}}<\infty
$$

and $A \in\left(l(\mu), l_{\infty}\right)$ iff $(b)$ holds.

(v) $A \in\left(l(\mu), c_{0}\right)$ iff $A \in\left(l(\mu), l_{\infty}\right)$ and $\lim _{n \rightarrow \infty} a_{n v}=0$ for every $v \in \mathbb{N}$.

Note that the condition (2.7) has some difficulties in applications. The following lemma presents a more useful and equivalent condition to (2.7). 
Lemma 2.2. ([26]) Let $\left(\mu_{v}\right)$ be a bounded sequence of positive numbers and $A=$ $\left(a_{n v}\right)$ be an infinite matrix with complex numbers. If $U_{\mu}[A]<\infty$ or $L_{\mu}[A]<\infty$, then

$$
(2 C)^{-2} U_{\mu}[A] \leq L_{\mu}[A] \leq U_{\mu}[A],
$$

where $C=\max \left\{1,2^{H-1}\right\}, H=\sup _{v} \mu_{v}$,

$$
U_{\mu}[A]=\sum_{v=0}^{\infty}\left(\sum_{n=0}^{\infty}\left|a_{n v}\right|\right)^{\mu_{v}}
$$

and

$$
L_{\mu}[A]=\sup \left\{\sum_{v=0}^{\infty}\left|\sum_{n \in K} a_{n v}\right|^{\mu_{v}}: K \subset \mathbb{N} \text { finite }\right\}
$$

Lemma 2.3. [22] Let $T$ be a triangle matrix, and let $X, Y$ be arbitrary subsets of $\omega$. Then, $A \in\left(X, Y_{T}\right)$ if and only if $B=T A \in(X, Y)$.

Lemma 2.4. [21, Theorem 3.9] Let $X$ be an FK space with $A K, T$ be a triangle matrix, $S$ be its inverse and $Y$ be an arbitrary subset of $\omega$. Then, we have $A \in$ $\left(X_{T}, Y\right)$ if and only if $\tilde{A}=\left(\tilde{a}_{n v}\right) \in(X, Y)$ and $V^{(n)}=\left(v_{m v}^{(n)}\right) \in(X, c)$ for all $n$, where

$$
\tilde{a}_{n v}=\sum_{j=v}^{\infty} a_{n j} s_{j v} ; \quad n, v=0,1, \ldots
$$

and

$$
v_{m v}^{(n)}=\left\{\begin{array}{cl}
\sum_{j=v}^{m} a_{n j} s_{j v}, & 0 \leq v \leq m \\
0, & v>m .
\end{array}\right.
$$

We begin with theorems by giving toplogical and algebraic structures of $\left|\mathcal{L}^{\phi}(r, s)\right|(\mu)$.

Theorem 2.1. Assume that $\left(\phi_{n}\right)$ is a sequence of positive numbers and $\left(\mu_{n}\right)$ is a bounded sequence of positive numbers.

(i) The set $\left|\mathcal{L}^{\phi}(r, s)\right|(\mu)$ is a linear space with coordinate-wise addition and scalar multiplication. Moreover, it is an FK-space with respect to the paranorm

$$
\|x\|_{\left|\mathcal{L}^{\phi}(r, s)\right|(\mu)}=\left\|E^{(\mu)} \circ \mathcal{L}(r, s)(x)\right\|_{l(\mu)},
$$

where $M=\max \left\{1, \sup _{n} \mu_{n}\right\}$. 
(ii) The sequence $b^{(j)}=\left(b_{n}^{(j)}\right)$ is a Schauder basis for the space $\left|\mathcal{L}^{\phi}(r, s)\right|(\mu)$, where

$$
b_{n}^{(j)}=\left\{\begin{array}{cl}
\phi_{j}^{\frac{-1}{\mu_{j}^{*}}}\left(\frac{1}{r} \frac{L_{n}}{L_{n}-1}+\sum_{k=j}^{n-1} \frac{(-1)^{n-k}}{r}\left(\frac{s}{r}\right)^{n-1-k}\right. & 1 \leq j \leq n-1 \\
\left.\cdot \frac{1}{L_{k} L_{k-1}}\left(\frac{s}{r} L_{n}^{2}+L_{n-1}^{2}\right)\right), & \\
\phi_{n}^{-1 / \mu_{n}^{*}} \frac{1}{r} \frac{L_{n}}{L_{n}-1}, & j=n \\
0, & j>n,
\end{array}\right.
$$

(iii) The space $\left|\mathcal{L}^{\phi}(r, s)\right|(\mu)$ is isometrically isomorphic to $l(\mu)$, i.e., $\left|\mathcal{L}^{\phi}(r, s)\right|(\mu) \cong l(\mu)$.

Proof. ( $i$ ) It is a routine verification to prove that $\left|\mathcal{L}^{\phi}(r, s)\right|(\mu)$ is a linear space, so we omit it. Further, since the space $l(\mu)$ is an $F K$-space and $E^{(\mu)} \circ \mathcal{L}(r, s)$ is a triangle matrix, it follows from Theorem 4.3 .2 of $[30],\left|\mathcal{L}^{\phi}(r, s)\right|(\mu)=(l(\mu))_{E^{(\mu)} \circ \mathcal{L}(r, s)}$ is an $F K$-space.

(ii) It is well-known that the sequence $\left(e^{(j)}\right)$ is the Schauder basis of the space $l(\mu)$. Also, since $b^{(j)}=\tilde{\mathcal{L}}(r, s)\left(\tilde{E}^{(\mu)}\left(e^{(j)}\right)\right)$, it is easily seen from Lemma 1.1 that the sequence $\left(b^{(j)}\right)$ is a Schauder basis of the space $\left|\mathcal{L}^{\phi}(r, s)\right|(\mu)$.

(iii) To prove this part, we must show that there exists a linear operator between these spaces which is bijective and norm-preserving. Now, consider the maps $\mathcal{L}(r, s):\left|\mathcal{L}^{\phi}(r, s)\right|(\mu) \rightarrow(l(\mu))_{E^{(\mu)}}$ and $E^{(\mu)}:(l(\mu))_{E^{(\mu)}} \rightarrow l(\mu)$ defined by the matrices (2.2) and (2.3). Since these matrices are triangles, the corresponding maps are bijection linear operator. Thus, the composite function $E^{(\mu)} \circ \mathcal{L}(r, s)$ is also a linear bijective operator. Further, by considering

$$
\|x\|_{\left|\mathcal{L}^{\phi}(r, s)\right|(\mu)}=\left\|E^{(\mu)} \circ \mathcal{L}(r, s)(x)\right\|_{l(\mu)},
$$

one can see that the composite function is norm-preserving. This completes the proof of the theorem. 
At this point, we list the following notations:

$$
\begin{aligned}
& \eta_{n j}=\frac{1}{r} \frac{L_{n}}{L_{n-1}}+\sum_{k=j}^{n-1} \frac{(-1)^{n-k}}{r}\left(\frac{s}{r}\right)^{n-1-k} \frac{1}{L_{k} L_{k-1}}\left(\frac{s}{r} L_{n}^{2}+L_{n-1}^{2}\right) \\
& D_{1}=\left\{\epsilon \in \omega: \sum_{n=j+1}^{\infty} \eta_{n j} \epsilon_{n} \text { exist for all } j\right\} \\
& D_{2}=\left\{\epsilon \in \omega: \exists M>1, \sup _{m}\left(\frac{M^{-1 / \mu_{m}^{*}}}{\phi_{m}}\left|\frac{1}{r} \frac{L_{m}}{L_{m-1}} \epsilon_{m}\right|^{\mu_{m}^{*}}\right.\right. \\
& \left.\left.+\sum_{j=1}^{m-1} \frac{M^{-1 / \mu_{j}^{*}}}{\phi_{j}}\left|\frac{1}{r} \frac{L_{j}}{L_{j-1}} \epsilon_{j}+\sum_{n=j+1}^{m} \eta_{n j} \epsilon_{n}\right|^{\mu_{j}^{*}}\right)<\infty\right\} \\
& D_{3}=\left\{\epsilon \in \omega: \sup _{m, j}\left(\left|\phi_{m}^{-1 / \mu_{m}^{*}} \frac{1}{r} \frac{L_{m}}{L_{m-1}} \epsilon_{m}\right|^{\mu_{m}}\right.\right. \\
& \left.\left.+\left|\phi_{j}^{-1 / \mu_{j}^{*}}\left(\frac{1}{r} \frac{L_{j}}{L_{j-1}} \epsilon_{j}+\sum_{n=j+1}^{m} \eta_{n j} \epsilon_{n}\right)\right|^{\mu_{j}}\right)<\infty\right\} \\
& D_{4}=\left\{\epsilon \in \omega: \exists M>1, \sum_{j=1}^{\infty} \frac{M^{-1 / \mu_{j}^{*}}}{\phi_{j}}\left(\sum_{n=j+1}^{\infty}\left|\eta_{n j} \epsilon_{n}\right|+\left|\frac{1}{r} \frac{L_{j}}{L_{j-1}} \epsilon_{j}\right|\right)^{\mu_{j}^{*}}<\infty\right\} \\
& D_{5}=\{\epsilon \in \omega: \exists M>1, \\
& \left.\sup _{j}\left(M^{-1 / \mu_{j}} \phi_{j}^{-1 / \mu_{j}^{*}}\left(\sum_{n=j+1}^{\infty}\left|\eta_{n j} \epsilon_{n}\right|+\left|\frac{1}{r} \frac{L_{j}}{L_{j-1}} \epsilon_{j}\right|\right)\right)<\infty\right\} .
\end{aligned}
$$

Theorem 2.2. Let $\phi=\left(\phi_{n}\right)$ be a sequence of positive numbers and $\mu=\left(\mu_{n}\right)$ be a bounded sequence of positive numbers.

(i) If $0<\mu_{n} \leq 1$ for all $n \in \mathbb{N}$, then

$$
\left\{\left|\mathcal{L}^{\phi}(r, s)\right|(\mu)\right\}^{\alpha}=D_{5},\left\{\left|\mathcal{L}^{\phi}(r, s)\right|(\mu)\right\}^{\beta}=D_{1} \cap D_{3},\left\{\left|\mathcal{L}^{\phi}(r, s)\right|(\mu)\right\}^{\gamma}=D_{3} .
$$

(ii) If $1<\mu_{n}<\infty$ for all $n \in \mathbb{N}$, then

$$
\left\{\left|\mathcal{L}^{\phi}(r, s)\right|(\mu)\right\}^{\alpha}=D_{4},\left\{\left|\mathcal{L}^{\phi}(r, s)\right|(\mu)\right\}^{\beta}=D_{1} \cap D_{2},\left\{\left|\mathcal{L}^{\phi}(r, s)\right|(\mu)\right\}^{\gamma}=D_{2} .
$$


Proof. Since the proof of the other parts are similar, to avoid repetition we only calculate the $\beta$-dual of the space $\left|\mathcal{L}^{\phi}(r, s)\right|(\mu)$. Let $x \in\left|\mathcal{L}^{\phi}(r, s)\right|(\mu)$. Note that $\epsilon \in$ $\left\{\left|\mathcal{L}^{\phi}(r, s)\right|(\mu)\right\}^{\beta}$ if $\epsilon x=\left(\epsilon_{n} x_{n}\right) \in c s$ for all $x \in\left|\mathcal{L}^{\phi}(r, s)\right|(\mu)$. Say $\mathcal{L}(r, s)(x)=y$ and $z=E^{(\mu)}(y)$. Then, since $\left|\mathcal{L}^{\phi}(r, s)\right|(\mu) \simeq l(\mu)$ by Theorem 2.1, $x \in\left|\mathcal{L}^{\phi}(r, s)\right|(\mu)$ if $z \in l(\mu)$, and so it is easily seen that

$$
\begin{aligned}
\sum_{n=1}^{m} \epsilon_{n} x_{n}= & \epsilon_{1} x_{1}+\sum_{n=2}^{m} \epsilon_{n}\left(\frac{1}{r} \frac{L_{n}}{L_{n-1}} y_{n}\right. \\
& \left.+\sum_{k=1}^{n-1} \frac{(-1)^{n-k}}{r}\left(\frac{s}{r}\right)^{n-1-k} \frac{1}{L_{k} L_{k-1}}\left(\frac{s}{r} L_{n}^{2}+L_{n-1}^{2}\right) y_{k}\right) \\
= & \sum_{j=1}^{m} \phi_{j}^{-1 / \mu_{j}^{*}} \sum_{n=j}^{m} \epsilon_{n} \frac{1}{r} \frac{L_{n}}{L_{n-1}} z_{j} \\
& +\sum_{j=1}^{m-1} \phi_{j}^{-1 / \mu_{j}^{*}}\left(\sum_{n=j+1}^{m} \sum_{k=j}^{n-1} \epsilon_{n} \frac{(-1)^{n-k}}{r}\left(\frac{s}{r}\right)^{n-1-k}\right. \\
& \left.\cdot \frac{1}{L_{k} L_{k-1}}\left(\frac{s}{r} L_{n}^{2}+L_{n-1}^{2}\right)\right) z_{j} \\
= & \phi_{m}^{-1 / \mu_{m}^{*}} \epsilon_{m} \frac{1}{r} \frac{L_{m}}{L_{m-1}} z_{m}+\sum_{j=1}^{m-1} \phi_{j}^{-1 / \mu_{j}^{*}}\left(\epsilon_{j} \frac{1}{r} \frac{L_{j}}{L_{j-1}}+\sum_{n=j+1}^{m} \epsilon_{n} \eta_{n j}\right) z_{j} \\
= & \sum_{j=1}^{m} b_{m j} z_{j}
\end{aligned}
$$

where $B=\left(b_{m j}\right)$ is the matrix defined by

$$
b_{m j}=\left\{\begin{array}{lr}
\phi_{j}^{-1 / \mu_{j}^{*}}\left(\epsilon_{j} \frac{1}{r} \frac{L_{j}}{L_{j-1}}+\sum_{n=j+1}^{m} \epsilon_{n} \eta_{n j}\right), & 1 \leq j \leq m-1 \\
\phi_{m}^{-1 / \mu_{m}^{*}} \epsilon_{m} \frac{1}{r} \frac{L_{m}}{L_{m-1}}, & j=m \\
0, & j>m .
\end{array}\right.
$$

This means that $\epsilon \in\left\{\left|\mathcal{L}^{\phi}(r, s)\right|(\mu)\right\}^{\beta}$ if and only if $B \in(l(\mu), c)$. Thus, by applying Lemma 2.1 to the matrix $B$, we obtain $\left\{\left|\mathcal{L}^{\phi}(r, s)\right|(\mu)\right\}^{\beta}=D_{1} \cap D_{2}$, for $1<\mu_{n}<\infty$, and $\left\{\left|\mathcal{L}^{\phi}(r, s)\right|(\mu)\right\}^{\beta}=D_{1} \cap D_{3}$, for $\mu_{n} \leq 1(n=0,1, \ldots)$, which completes the proof.

The following theorems show that certain matrix transformations on the space $\left|\mathcal{L}^{\phi}(r, s)\right|(\mu)$ correspond to bounded linear operators, and give their characterizations. 
Theorem 2.3. Let $\phi=\left(\phi_{n}\right)$ be a sequence of positive numbers, $\mu=\left(\mu_{n}\right)$ be bounded sequence of positive numbers, $A=\left(a_{n k}\right)$ be an infinite matrix of complex numbers for all $n, v \in \mathbb{N}$ and $B=\left(b_{n k}\right)$ be a matrix satisfying the following relation

$$
b_{n k}=\phi_{n}^{1 / \mu_{n}^{*}} \sum_{v=0}^{n} \xi_{n v} a_{v k} .
$$

Then, for any sequence spaces $\lambda, A \in\left(\lambda,\left|\mathcal{L}^{\phi}(r, s)\right|(\mu)\right)$ if and only if $B \in(\lambda, l(\mu))$.

Proof. Take $x \in \lambda$. It follows from (2.8) that

$$
\sum_{k=0}^{\infty} b_{n k} x_{k}=\phi_{n}^{1 / \mu_{n}^{*}} \sum_{v=0}^{n} \xi_{n v} \sum_{k=0}^{\infty} a_{v k} x_{k} .
$$

By definition of $\xi$, it is seen immediately that $B_{n}(x)=\left(E^{(\mu)} \circ \mathcal{L}(r, s)\right)_{n}(A(x))$ for all $x \in \lambda$. So, it is obtained that $A_{n}(x) \in\left|\mathcal{L}^{\phi}(r, s)\right|(\mu)$ whenever $x \in \lambda$ if and only if $B(x) \in l(\mu)$ whenever $x \in \lambda$, which completes the proof of the theorem.

Theorem 2.4. Assume that $\left(\phi_{n}\right)$ and $\left(\psi_{n}\right)$ are sequences of positive numbers, and $\left(\mu_{n}\right)$ and $\left(\lambda_{n}\right)$ are bounded sequences of positive numbers with $\mu_{n} \leq 1$ and $\lambda_{n} \geq 1$. Further, let $A=\left(a_{n k}\right)$ be an infinite matrix of complex numbers for all $n, k \in \mathbb{N}$ and $\hat{A}^{(\lambda)}=E^{(\lambda)} \circ \mathcal{L}(r, s) \circ \tilde{A}$, where

$$
\tilde{a}_{n v}=\phi_{v}^{-1 / \mu_{v}^{*}}\left(\frac{1}{r} \frac{L_{v}}{L_{v-1}} a_{n v}+\sum_{j=v+1}^{\infty} a_{n j} \eta_{j v}\right) .
$$

If $A \in\left(\left|\mathcal{L}^{\phi}(r, s)\right|(\mu),\left|\mathcal{L}^{\psi}(r, s)\right|(\lambda)\right)$, then $A$ defines a bounded linear operator $L_{A}$ such that $L_{A}(x)=A(x)$ for all $x \in\left|\mathcal{L}^{\phi}(r, s)\right|(\mu)$, and $A \in\left(\left|\mathcal{L}^{\phi}(r, s)\right|(\mu)\right.$, $\left.\left|\mathcal{L}^{\psi}(r, s)\right|(\lambda)\right)$ if and only if there exists an integer $M>0$ such that, for all $n$,

$$
\begin{gathered}
\sum_{v=j+1}^{\infty} \eta_{v j} a_{n v} \text { exists for all } j \\
\sup _{m, k}\left\{\left|\phi_{m}^{-1 / \mu_{m}^{*}} \frac{1}{r} \frac{L_{m}}{L_{m-1}} a_{n m}\right|^{\mu_{m}}\right. \\
\left.+\left(\phi_{k}^{-1 / \mu_{k}^{*}}\left|\frac{1}{r} \frac{L_{k}}{L_{k-1}} a_{n k}+\sum_{j=k+1}^{m} \eta_{j k} a_{n j}\right|\right)^{\mu_{k}}\right\}<\infty \\
\sup _{v} \sum_{n=0}^{\infty}\left|M^{-1 / \mu_{v}} \hat{a}_{n v}^{(\lambda)}\right|^{\lambda_{n}}<\infty
\end{gathered}
$$


Proof. By Theorem 2.1, the spaces $\left|\mathcal{L}^{\phi}(r, s)\right|(\mu)$ and $\left|\mathcal{L}^{\psi}(r, s)\right|(\lambda)$ are $F K$-spaces. Thus, by Theorem 4.2.8 of [30], $L_{A}$ is a bounded linear operator.

To prove the second part, take $A \in\left(\left|\mathcal{L}^{\phi}(r, s)\right|(\mu),\left|\mathcal{L}^{\psi}(r, s)\right|(\lambda)\right)$. Then, by Lemma $2.4, \tilde{A} \in\left(l(\mu),\left|\mathcal{L}^{\psi}(r, s)\right|(\lambda)\right)$ and $V^{(n)} \in(l(\mu), c)$, where $V^{(n)}$ is the matrix given by

$$
v_{m k}^{(n)}=\left\{\begin{array}{lr}
\phi_{k}^{-1 / \mu_{k}^{*}}\left(a_{n k} \frac{1}{r} \frac{L_{k}}{L_{k-1}}+\sum_{j=k+1}^{m} a_{n j} \eta_{j k}\right), & 0 \leq k \leq m-1 \\
\phi_{m}^{-1 / \mu_{m}^{*}} a_{n m \frac{1}{r} \frac{L_{m}}{L_{m-1}},} & k=m \\
0, & k>m .
\end{array}\right.
$$

Applying the Lemma 2.1 to the matrix $V^{(n)}$, we have the conditions (2.9) and (2.10). Also, for $x \in l(\mu)$, it follows from $\left|\mathcal{L}^{\psi}(r, s)\right|(\lambda)=\{l(\lambda)\}_{E^{(\lambda)} \circ \mathcal{L}(r, s)}$ that $\tilde{A}(x) \in\left|\mathcal{L}^{\psi}(r, s)\right|(\lambda)$ if and only if $\hat{A}^{(\lambda)}(x)=E^{(\lambda)} \circ \mathcal{L}(r, s) \circ \tilde{A}(x) \in l(\lambda)$. This gives that $\tilde{A} \in\left(l(\mu),\left|\mathcal{L}^{\psi}(r, s)\right|(\lambda)\right)$ iff $\hat{A}^{(\lambda)} \in(l(\mu), l(\lambda))$. So, the proof is completed together with Lemma 2.1 .

Theorem 2.5. Let $\left(\phi_{n}\right)$ and $\left(\psi_{n}\right)$ be sequences of positive numbers, and $\left(\mu_{n}\right)$ be bounded sequence of positive numbers with $\mu_{n}>1$. Also, let $A=\left(a_{n k}\right)$ be an infinite matrix of complex numbers for each $n, k \in \mathbb{N}$. Define the matrix $\hat{A}^{(1)}=E^{(1)} \circ \mathcal{L}(r, s) \circ$ $\tilde{A}$, where $\tilde{A}$ is as in Theorem 2.4. If $A \in\left(\left|\mathcal{L}^{\phi}(r, s)\right|(\mu),\left|\mathcal{L}^{\psi}(r, s)\right|\right)$, then $A$ defines a bounded linear operator $L_{A}$ such that $L_{A}(x)=A(x)$ for all $x \in\left|\mathcal{L}^{\phi}(r, s)\right|(\mu)$. Also, $A \in\left(\left|\mathcal{L}^{\phi}(r, s)\right|(\mu),\left|\mathcal{L}^{\psi}(r, s)\right|\right)$ if and only if there exists an integer $M>1$ such that, for all $n \in \mathbb{N}$,

$$
\begin{gathered}
\sup _{m}\left\{\frac{M^{-1 / \mu_{m}^{*}}}{\phi_{m}}\left|\frac{1}{r} \frac{L_{m}}{L_{m-1}} a_{n m}\right|^{\mu_{m}^{*}}\right. \\
\left.+\sum_{j=1}^{m-1} \frac{M^{-1 / \mu_{j}^{*}}}{\phi_{j}}\left|\frac{1}{r} \frac{L_{j}}{L_{j-1}} a_{n j}+\sum_{v=j+1}^{m} \eta_{v j} a_{n v}\right|^{\mu_{j}^{*}}\right\}<\infty, \\
\sum_{v=1}^{\infty}\left(\sum_{n=1}^{\infty}\left|\hat{a}_{n v} M^{-1}\right|\right)^{\mu_{v}^{*}}<\infty .
\end{gathered}
$$

Proof. The first part is proved as before. Also, since $\left|\mathcal{L}^{\phi}(r, s)\right|(\mu)=(l(\mu))_{E^{(\mu)} \circ \mathcal{L}(r, s)}$, by Lemma 2.4, $A \in\left(\left|\mathcal{L}^{\phi}(r, s)\right|(\mu),\left|\mathcal{L}^{\psi}(r, s)\right|\right)$ iff $\tilde{A} \in\left(l(\mu),\left|\mathcal{L}^{\phi}(r, s)\right|\right)$ and $V^{(n)} \in$ 
$(l(\mu), c)$. Further, by Lemma 2.1, $V^{(n)} \in(l(\mu), c)$ iff the conditions $(2.12)$ and (2.13) hold, and $\tilde{A} \in\left(l(\mu),\left|\mathcal{L}^{\phi}(r, s)\right|\right)$ iff $\hat{A}^{(1)}=E^{(1)} \circ \mathcal{L}(r, s) o \tilde{A} \in(l(\mu), l)$, which completes the proof applying Lemma 2.1 to the matrix $\hat{A}^{(1)}$.

By following the above lines, we also have the following.

Theorem 2.6. Let $\left(\phi_{n}\right)$ be a sequence of positive numbers, and $\left(\mu_{n}\right)$ be a bounded sequences of positive numbers. Further let $A=\left(a_{n k}\right)$ be an infinite matrix of complex numbers for all $n, k \in \mathbb{N}$ and $Y$ be arbitrary sequence space. Then, $A \in$ $\left(\left|\mathcal{L}^{\phi}(r, s)\right|(\mu), Y\right)$ if and only if

$$
\begin{gathered}
V^{(n)} \in(l(\mu), c) \text { for all } n \in \mathbb{N}, \\
\tilde{A} \in(l(\mu), Y),
\end{gathered}
$$

where the matrices $V^{(n)}$ and $\tilde{A}$ are as in Theorem 2.4.

Now, we list the following notations:

(i) $\sup _{n, k}\left|\tilde{a}_{n k}\right|^{\mu_{k}}<\infty$.

(ii) There exists $M>1$ such that $\sup _{n} \sum_{k}\left|M^{-1} \tilde{a}_{n k}\right|^{\mu_{k}^{*}}<\infty$.

(iii) $\lim _{n \rightarrow \infty} \tilde{a}_{n k}=0$ for each $k \in \mathbb{N}$.

(iv) $\quad \lim _{n \rightarrow \infty} \tilde{a}_{n k}$ exists for all $k \in \mathbb{N}$.

(v) There exists $M>1$ such that $\sup _{k} \sum_{n=0}^{\infty}\left|M^{-1 / \mu_{k}} \tilde{a}_{n k}\right|<\infty$.

(vi) There exists $M>1$ such that

$$
\sup \left\{\sum_{k=0}^{\infty}\left|\sum_{n \in K} \tilde{a}_{n k} M^{-1}\right|^{\mu_{k}^{*}}: K \subset \mathbb{N} \text { finite }\right\}<\infty .
$$

(vii) $\sup _{m, k}\left|v_{m k}^{(n)}\right|^{\mu_{k}}<\infty$.

(viii) There exists $M>1$ such that $\sup _{m} \sum_{k}\left|M^{-1} v_{m k}^{(n)}\right|^{\mu_{k}^{*}}<\infty$.

(ix) $\lim _{m \rightarrow \infty} v_{m k}^{(n)}$ exists for all $n, k \in \mathbb{N}$.

Thus, by combining our theorems with Lemma 2.1 we obtain the following results:

Theorem 2.7. The following statements hold:

1. If $\mu_{n} \leq 1$ for all $n$, then, $A \in\left(\left|\mathcal{L}^{\phi}(r, s)\right|(\mu), l_{\infty}\right) \Leftrightarrow$ (i), (vii) and (ix) hold. 
2. If $\mu_{n}>1$ for all $n$, then, $A \in\left(\left|\mathcal{L}^{\phi}(r, s)\right|(\mu), l_{\infty}\right) \Leftrightarrow$ (ii), (viii) and (ix) hold.

3. If $\mu_{n} \leq 1$ for all $n$, then, $A \in\left(\left|\mathcal{L}^{\phi}(r, s)\right|(\mu), c\right) \Leftrightarrow$ (i), (iv), (vii) and (ix) hold.

4. If $\mu_{n}>1$ for all $n$, then, $A \in\left(\left|\mathcal{L}^{\phi}(r, s)\right|(\mu), c\right) \Leftrightarrow$ (ii), (iv), (viii) and (ix) hold.

5. If $\mu_{n} \leq 1$ for all $n$, then, $A \in\left(\left|\mathcal{L}^{\phi}(r, s)\right|(\mu), c_{0}\right) \Leftrightarrow$ (i), (iii), (vii) and (ix) hold.

6. If $\mu_{n}>1$ for all $n$, then, $A \in\left(\left|\mathcal{L}^{\phi}(r, s)\right|(\mu), c_{0}\right) \Leftrightarrow$ (ii), (iii), (viii) and (ix) hold.

7. If $\mu_{n} \leq 1$ for all $n$, then, $A \in\left(\left|\mathcal{L}^{\phi}(r, s)\right|(\mu), l\right) \Leftrightarrow$ (v), (vii) and (ix) hold.

8. If $\mu_{n}>1$ for all $n$, then, $A \in\left(\left|\mathcal{L}^{\phi}(r, s)\right|(\mu), l\right) \Leftrightarrow$ (vi), (viii) and (ix) hold.

Also, Theorem 2.7 gives the following.

Corollary 2.1. Put $a(n, k)=\sum_{j=0}^{n} a_{j k}$ instead of $a_{n k}$ for all $n, k$.Then,

1. If $\mu_{n} \leq 1$ for all $n$, then, $A \in\left(\left|\mathcal{L}^{\phi}(r, s)\right|(\mu), b s\right) \Leftrightarrow$ (i), (vii) and (ix) hold.

2. If $\mu_{n}>1$ for all $n$, then, $A \in\left(\left|\mathcal{L}^{\phi}(r, s)\right|(\mu), b s\right) \Leftrightarrow$ (ii), (viii) and (ix) hold.

3. If $\mu_{n} \leq 1$ for all $n$, then, $A \in\left(\left|\mathcal{L}^{\phi}(r, s)\right|(\mu), c s\right) \Leftrightarrow$ (i), (iv), (vii) and (ix) hold.

4. If $\mu_{n}>1$ for all $n$, then, $A \in\left(\left|\mathcal{L}^{\phi}(r, s)\right|(\mu), c s\right) \Leftrightarrow$ (ii), (iv), (viii) and (ix) hold.

\section{RE F E R E N C E S}

1. B. Altay and F. BAŞAR: Some paranormed Riesz sequence spaces of non-absolute type. Southeast Asian Bull. Math. 30(5) (2006), 591-608.

2. H. Bilgin and S. Demiriz: Some Algebraic And Topological Properties Of New Lucas Difference Sequence Spaces. Turk. J. Math. 10 (Special Issue: Proceedings of ICMME 2018), 144-152.

3. H. Bor: On $\left|\bar{N}, p_{n}\right|_{k}$ summability factors of infinite series. Tamkang J. Math. 16 (1985), 13-20.

4. S. Demiriz and C.ÇAKan: Some New Paranormed Difference Sequence Spaces And Weighted Core. Comput. Math. with Appl. 64(6) (2012), 1726-1739.

5. T. M. FLETT: On an extension of absolute summability and some theorems of Littlewood and Paley. Proc. Lond. Math. Soc. 7(1957), 113-141. 
6. F. GöKÇE and M. A. SARIGÖL: A new series space $\left|\bar{N}_{p}^{\theta}\right|(\mu)$ and matrix transformations with applications. Kuwait J. Sci. 45(4) (2018), 1-8.

7. F. GÖKÇE and M. A. SARIGÖL: Generalization of the space $l(p)$ derived by absolute Euler summability and matrix operators. J. Inequal. Appl. 2018 (1) (2018), 133.

8. F. GÖKÇE and M. A. SARIGÖL: Generalization of the absolute Cesàro space and some matrix transformations. Numer. Funct. Anal. Optim. 40(9) (2019), 1039-1052.

9. F. GöKÇE and M. A. SARIGÖL: Some matrix and compact operators of the absolute Fibonacci series spaces. Kragujevac J. Math. 44(2) (2020), 273-286.

10. M. Ilkhan, S. Demiriz and E. E. Kara: A New Paranormed Sequence Space Defined by Euler Totient Matrix. Karaelmas Sci. Eng. J. 9(2) (2019), 277-282.

11. A. M. JARRAH and E. MALKOWSKY: Ordinary absolute and strong summability and matrix transformations. Filomat. 17 (2003), 59-78.

12. K. G. Grosse-Erdmann: Matrix transformations between the sequence spaces of Maddox. J. Math. Anal. Appl. 180 (1993), 223-238.

13. E. E. KARA and M. ILKHAN : Some properties of generalized Fibonacci sequence spaces. Linear Multilinear Algebra. 64(11) (2016), 2208-2223.

14. E. E. Kara and S. Demiriz: Some New Paranormed Difference Sequence Spaces Derived By Fibonacci Numbers. Miskolc Math. Notes. 16(2) (2015), 907-923.

15. M. KARAKAŞ and A. M. KARAKAŞ: A study on Lucas difference sequence spaces $l_{p}(\hat{E}(r, s))$ and $l_{\infty}(\hat{E}(r, s))$. Maejo Int. J. Sci. Technol. 12(1) (2018), 70-78.

16. V. Karakaya, A. K. Noman and H. Polat: On paranormed $\lambda$-sequence spaces of non-absolute type. Math. Comp. Model. 54(5) (2011), 1473-1480.

17. T. Koshy: Fibonacci and Lucas numbers with applications. 51, John Wiley and Sons, 2011.

18. I. J. MADDOX: Some properties of paranormed sequence spaces. J. London Math. Soc. 2 (1969), 316-322.

19. I. J. MADDOX: Paranormed sequence spaces generated by infinite matrices. Math. Proc. Cambridge Philos. Soc. 64 (1968), 335-340.

20. I. J. MAdDox: Spaces of strongly summable sequences. Q. J. Math. 18 (1947), 345-355.

21. E. Malkowsky and V. Rakocevic: On matrix domains of triangles. Appl. Math. Comput. 189(2) (2007), 1146-1163.

22. E. Malkowsky and V. Rakocevic: An introduction into the theory of sequence space and measures of noncompactness. Zbornik radova (Beogr). 9(17) (2000), 143-234

23. M. Mursaleen: Applied Summability Methods. Springer, Heidelberg, 2013.

24. R. N. MohapatrA and M. A. SARIGÖL: On matrix operators on the series spaces $\left|\bar{N}_{p}^{\theta}\right|_{k}$. Ukrainian Math. J. 69(11) (2018), 1524-1533.

25. M. A. SARIGÖL: Spaces of Series Summable by Absolute Cesàro and matrix operators. Comm. Math Appl. 7(1) (2016), 11-22.

26. M. A. SARIGÖL: An inequality for matrix operators and its applications. J. Class. Anal. 2 (2013), 145-150.

27. M. A. SARIGÖL: Matrix transformations on fields of absolute weighted mean summability. Studia Sci. Math. Hungar. 48(3) (2011), 331-341.

28. M. A. SARIGÖL: On the local properties of factored Fourier series. Appl. Math. Comput. 216(11) (2010), 3386-3390. 
29. W. T. Sulaiman: On summability factors of infinite series. Proc. Amer. Math. Soc. 115 (1992), 313- 317.

30. A. Wilansky: Summability Through Functional Analysis, Mathematics Studies. 85. North Holland , Amsterdam, 1984. 